\title{
Hippocampal structural plasticity accompanies the resulting contextual fear memory following stress and fear conditioning
}

\author{
Marcelo Giachero, Gaston D. Calfa, ${ }^{1}$ and Victor A. Molina ${ }^{1}$ \\ IFEC-CONICET, Departamento de Farmacología, Facultad de Ciencias Químicas, Universidad Nacional de Córdoba, \\ (5000) Córdoba, Argentina
}

\begin{abstract}
The present research investigated the resulting contextual fear memory and structural plasticity changes in the dorsal hippocampus $(\mathrm{DH})$ following stress and fear conditioning. This combination enhanced fear retention and increased the number of total and mature dendritic spines in DH. Intra-basolateral amygdala (BLA) infusion of midazolam prior to stress prevented both the enhancement of fear retention and an increase in the density of total and mature dendritic spines in DH. These findings emphasize the role of the stress-induced attenuation of GABAergic neurotransmission in BLA in the promoting influence of stress on fear memory and on synaptic remodeling in DH. In conclusion, the structural remodeling in DH accompanied the facilitated fear memory following a combination of fear conditioning and stressful stimulation.
\end{abstract}

It is well accepted that prior exposure to stressful events facilitates the emergence of fear memory (Shors et al. 1992; Beylin and Shors 1998; Shors 2001; Cordero et al. 2003; Rodriguez Manzanares et al. 2005). Consistent with this, recent data shows that the interaction of an unrelated aversive experience and an established fear memory trace results in a robust and persistent fear memory (Giachero et al. 2013). This stress-induced promoting influence was prevented by midazolam (MDZ) intra-basolateral amygdala (BLA) infusion prior to stress, indicating that such an influence is related to the modulation of the GABAergic transmission in BLA (Giachero et al. 2013).

Dendritic spines represent the structural platform for excitatory synaptic contacts between neurons (Gray 1959; Chapleau and Pozzo-Miller 2007), with growing evidence suggesting that synaptic remodeling accompanies the formation of long-term memory (Restivo et al. 2009). In line with this view, the encoding of a novel contextual representation was shown to result in a rapid synaptic rearrangement through an increase in spine density, particularly in the hippocampus across multiple memory paradigms, including contextual fear memory (Leuner et al. 2003; Restivo et al. 2009; Vetere et al. 2011).

The dorsal hippocampus (DH) is crucially involved in the contextual representation following fear conditioning (Kim and Fanselow 1992; Phillips and LeDoux 1995; Maren and Fanselow 1997; Fanselow and Dong 2010). Related to this, the present research investigates the structural plasticity in the $\mathrm{DH}$ that may underlie the contextual fear memory resulting from the interaction of fear conditioning and an unrelated stressful experience. The BLA is an essential component of the neural circuitry orchestrating emotional response to threatening stimuli (LeDoux 2000), and compelling evidence has shown that the activation of GABAa sites, specifically in BLA prior to stress, attenuates the enhancement of fear memory (Rodriguez Manzanares et al. 2005; Giachero et al. 2013). Therefore, we also explored the modulatory role of the GABAergic neurotransmission in the BLA in both fear retention and structural plasticity in the $\mathrm{DH}$, which is

\footnotetext{
${ }^{1}$ Corresponding authors

E-mail vmolina@fcq.unc.edu.ar

E-mail gcalfa@fcq.unc.edu.ar

Article is online at http://www.learnmem.org/cgi/doi/10.1101/lm.031724.113.
}

potentially associated with the fear memory resulting from such an interaction.

The behavioral procedure used was similar to that previously described by our laboratory (Giachero et al. 2013). On the day of the experiment, two groups of rats (adult male Wistar rats, weighing between 280 and 320 g, from our vivarium) were randomly selected and individually placed in the conditioning chamber, denoted as CA. Following a 3-min acclimation period (pre-shock period), rats were either subjected to a single unsignaled footshock (0.3 mA; 3-sec duration) (CA-US), or exposed to the CA without the footshock (CA-noUS). All animals were kept in the chamber for an additional $50 \mathrm{sec}$ (post-shock period). Then, $24 \mathrm{~h}$ later, both groups of animals were once more randomly selected and either exposed to the restraint experience (30 min inside a plastic cylindrical restrainer [Stress, S] fitted close to the body, thus preventing animal movement except for the tail and the tip of the nose; Rodriguez Manzanares et al. 2005; Maldonado et al. 2011) or gently handled without any stressful manipulation (No Stress, NS). No other subjects were present in the experimental room during stress exposure. One day after this manipulation, a fear memory test was run by placing the animals in the CA without footshocks for $5 \mathrm{~min}$ to assess the freezing behavior (defined as a total absence of body and head movement except those associated with breathing), or animals were sacrificed for structural plasticity analysis. The interval between stress and behavioral testing or sacrifice was selected based on previous data from this laboratory using a similar stress protocol where it was reported that, following this period, stressed animals exhibited a relevant freezing behavior indicative of fear retention (Rodriguez Manzanares et al. 2005; Giachero et al. 2013).

In experiments where local BLA drug administration was required, rats were anesthetized with a mixture of ketamine (55 $\mathrm{mg} / \mathrm{kg}$, i.p., Ketaject) and xylazine (11 mg/kg i.p., Xyla-Ject) and

(C) 2013 Giachero et al. This article is distributed exclusively by Cold Spring Harbor Laboratory Press for the first 12 months after the fullissue publication date (see http://learnmem.cshlp.org/site/misc/terms. xhtml). After 12 months, it is available under a Creative Commons License (Attribution-NonCommercial 3.0 Unported), as described at http://creativecommons.org/licenses/by-nc/3.0/. 
placed in a stereotaxic instrument (Stoelting). The following coordinates aimed to BLA were used: anterior, $-2.8 \mathrm{~mm}$; lateral, \pm 5.0 $\mathrm{mm}$; ventral, $-6.1 \mathrm{~mm}$ (Fig. 2Cii, see below) (Paxinos and Watson 2007). Experiments started after a 7-d recovery period (Rodriguez Manzanares et al. 2005; Giachero et al. 2013).

For structural plasticity analysis, animals were deeply anesthetized with chloral hydrate $(400 \mathrm{mg} / \mathrm{kg}$ i.p.) before being perfused transcardially first by ice-cold $\mathrm{PB}(0.1 \mathrm{M}, \mathrm{pH} 7.4)$ and fixed using ice-cold $4 \%$ paraformaldehyde (dissolved in $0.1 \mathrm{M} \mathrm{PB}, \mathrm{pH}$ 7.4). The brain was removed and post-fixed in the same fixative for $24 \mathrm{~h}$ at $4^{\circ} \mathrm{C}$, and then sectioned with a vibratome $(200 \mu \mathrm{m}$ thick) to isolate brain slices containing the $\mathrm{DH}$, which were collected in PBS $0.1 \%$. The CA1 DH was stained with small droplets $(<10 \mu \mathrm{m})$ of a saturated solution of the lipophilic dye, 1, $1^{\prime}$ dioctadecyl-3,3,3',3'-tetramethyl indocarbocyanine perchlorate (DiI, InVitrogen) in fish oil (Pozzo-Miller et al. 1999) by microinjection with a patch pipette and positive pressure application (see below, Figs. 1C, 2Ci,Cii). After $24 \mathrm{~h}$ at room temperature in the dark, $z$-sections from labeled dendritic segments were collected using a Fluoview FV-300 laser-scanning confocal microscope (Olympus IX81 inverted microscope) with an oil immersion (NA 1.42) objective lens (PlanApo, Centro de Microscopía Óptica y Confocal de Avanzada.
The images were deconvolved using the "advanced maximum likelihood estimation algorithm" for Cell R software (Olympus Soft Imaging Solutions), version 3.3, set with 15 iterations and an overlay subvolume of 10 pixels. A theoretical point spread function was used. Dendritic projections shorter than $3 \mu \mathrm{m}$ were quantified manually (Murphy and Segal 1996; Chapleau et al. 2009; Calfa et al. 2012) using ImageJ software (National Institutes of Health) and a computer-assisted method, Neuron Studio software (Rodriguez et al. 2008), was also used for spine density and shape evaluation. Considering a high density of labeled dendrites, presumably from different neurons but from CA1 stratum radiatum, single segments from the $z$-section projections were used to count the total number and also the number of each particular type of dendritic spine normalized to $10 \mu \mathrm{m}$ of the dendritic segment length, ensuring that each spine was counted only once by following its course in the $z$-section reconstructions.

Spine types were classified as type-I or "stubby" shaped dendritic spines, type-II or "mushroom" shaped dendritic spines, and type-III or "thin" shaped dendritic spines, following previously published criteria (Koh et al. 2002; Tyler and Pozzo-Miller 2003; Boda et al. 2004). In brief, for each dendritic protrusion, different measurements were taken: the length (dimension from the base at

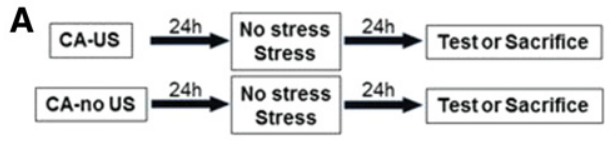

D

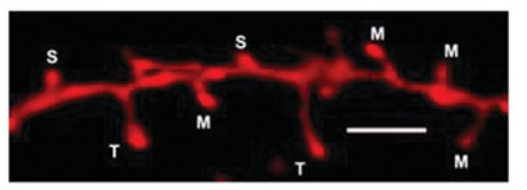

E

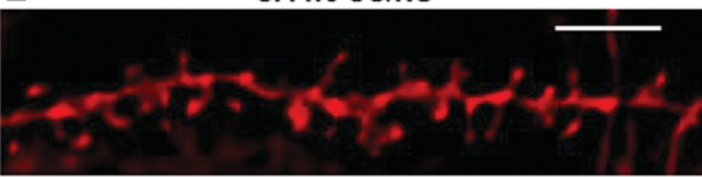

CA-US/NS
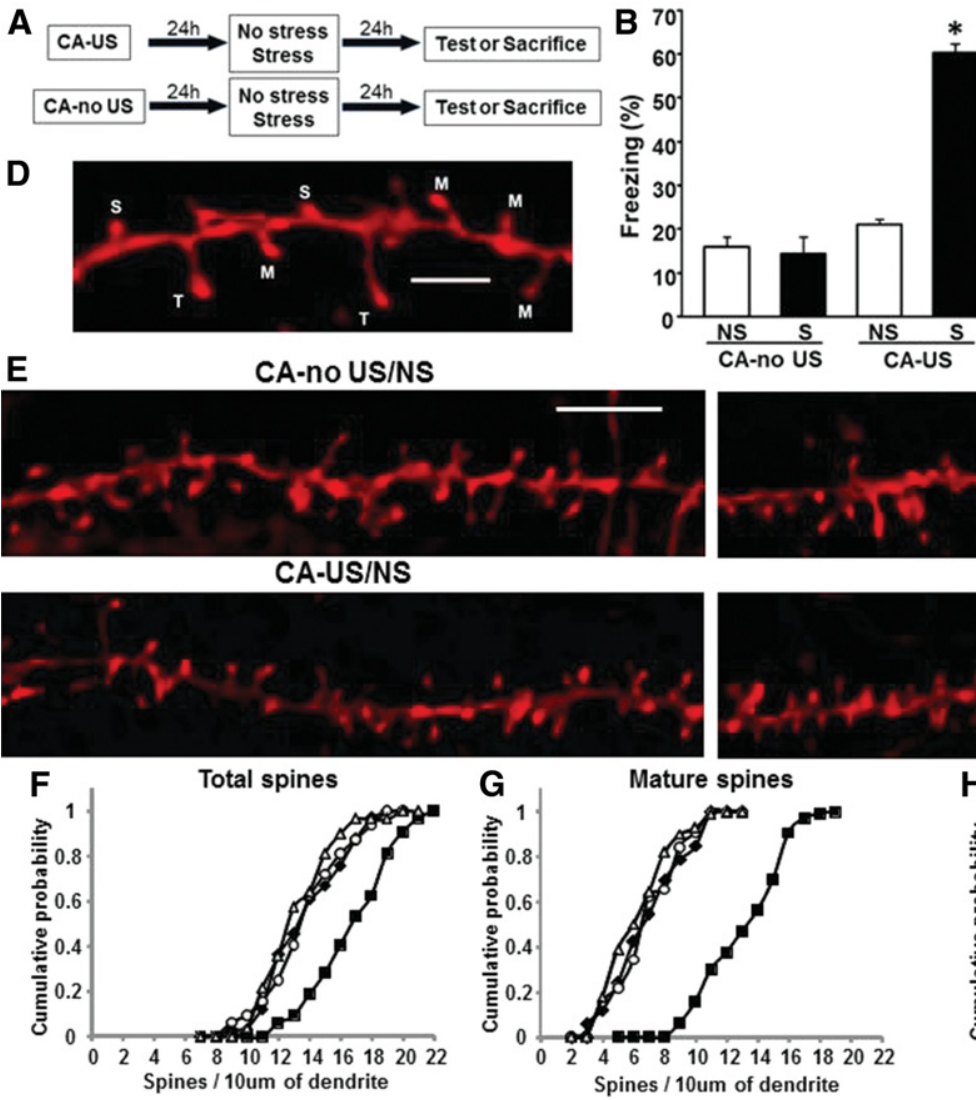

C
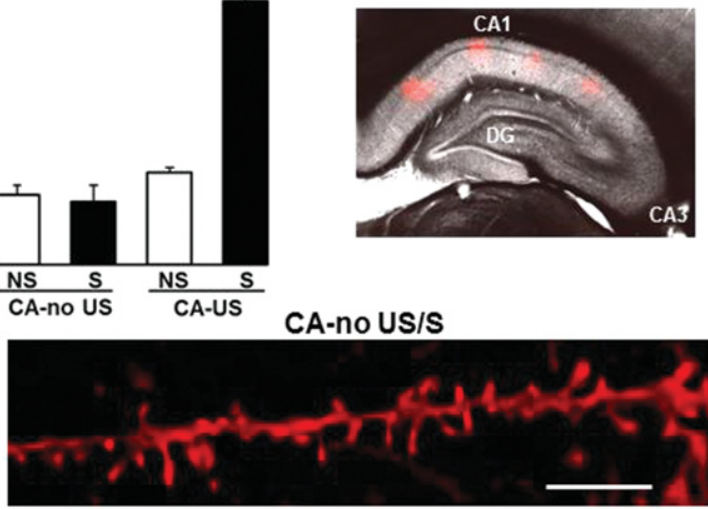

CA-US/S
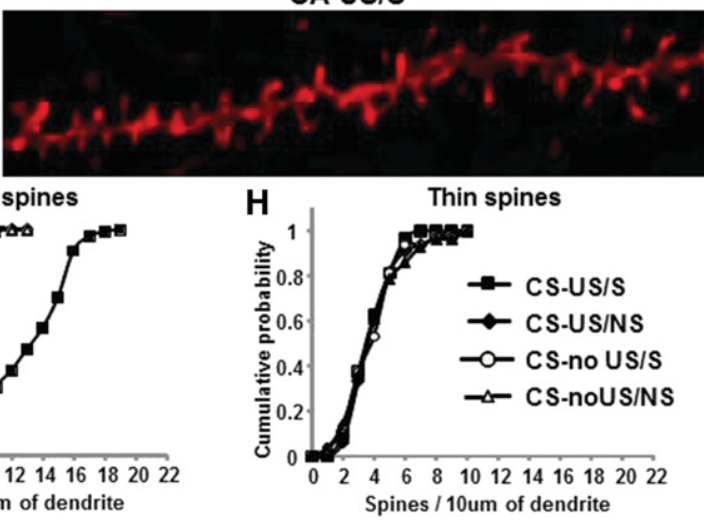

Figure 1. The combination between the conditioning procedure and the stress enhanced fear memory retention and generated $\mathrm{DH}$ structural changes. $(A)$ Schematic representation of the experimental design. (B) Bar graph showing the freezing response denoted in animals during the test session performed $1 \mathrm{~d}$ after the exposure to a stressful experience. Data are expressed as mean \pm SEM of the percentage of freezing spent during the test ( $n=8$ rats per group). $\left(^{*}\right) P<0.05$ compared to the rest of the experimental groups (two-way ANOVA, Bonferroni post-hoc test). (C) Bright field along with fluorescence image of a representative DH CA1 region acquired simultaneously in order to demonstrate the localization of the Dil labeling. (D) Representative example showing the morphology of the different types of dendritic spines observed: (M) mushroom-shaped dendritic spines, (S) stubbyshaped dendritic spines, (T) thin-shaped dendritic spines. Bar, $2 \mu \mathrm{m}$. (E) Representative examples of apical dendritic segments of CA1 pyramidal neurons which were selected for quantitative analysis of dendritic spines from animals: CA-noUS $/ \mathrm{NS}(n=3$ rats), CA-noUS $/ \mathrm{S}(n=3)$, CA-US/NS ( $n=3)$, and CA-US/S $(n=3)$. Bar, $3 \mu \mathrm{m}$. $(F-H)$ Cumulative frequency of total $(F)$, mature $(G)$, and thin $(H)$ spine density on apical dendrites of hippocampal pyramidal cells $C A 1$ stratum radiatum $(P<0.05$, by Kolmogorov-Smirnov test [KS test]). 


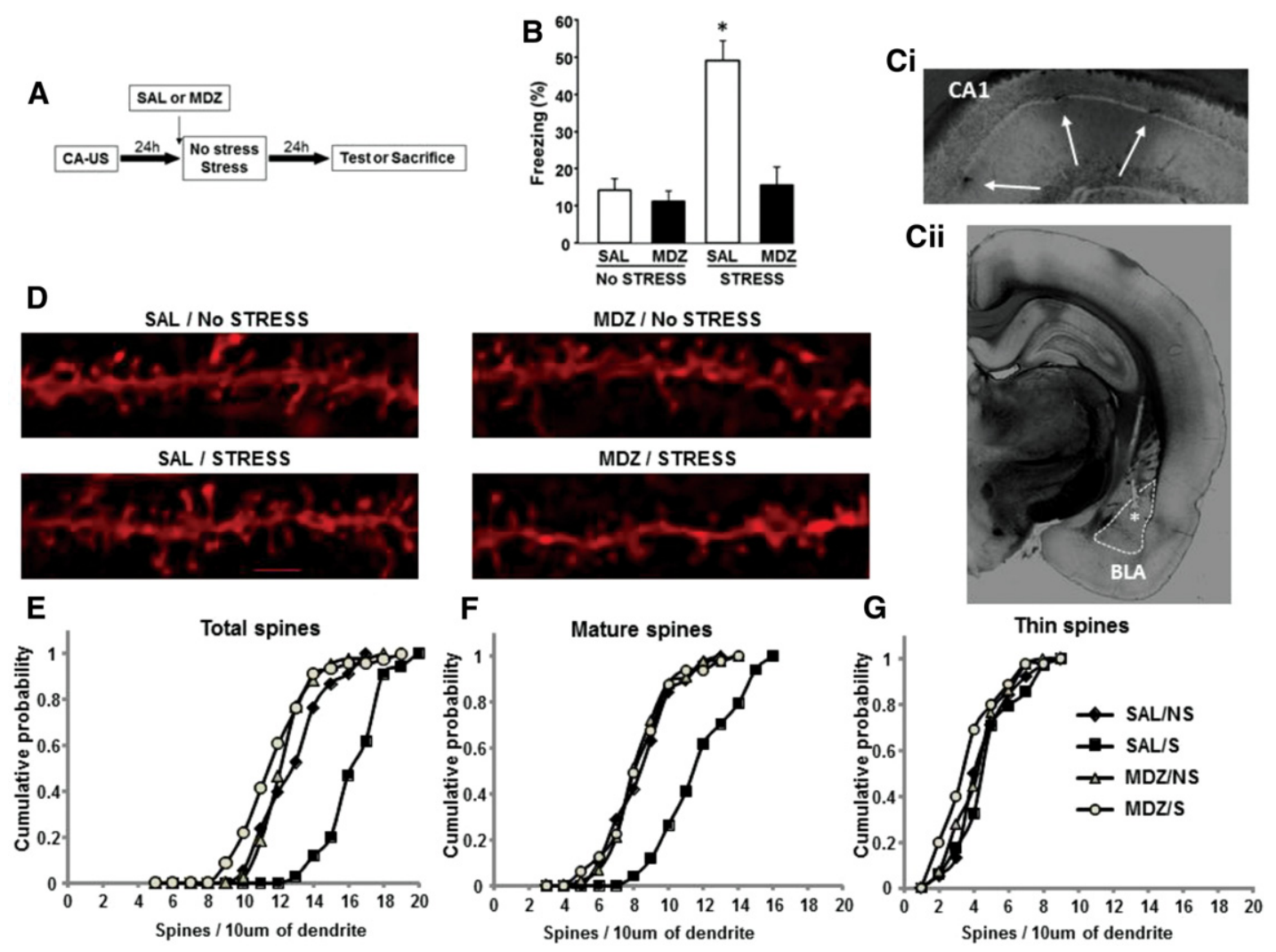

Figure 2. Intra-basolateral amygdala infusion of MDZ prior to stress attenuates the promoting influence of restraint on both fear retention and DH structural remodeling. (A) Schematic representation of the experimental design. (B) Freezing behavior response observed during testing in rats that received either intra-BLA MDZ or SAL administration 10 min prior to restraint. Data are expressed as mean \pm SEM of the percentage of freezing spent during the test ( $n=8$ rats for all groups). ( $\left.{ }^{*}\right) P<0.05$ compared to the rest of the experimental groups (two-way ANOVA, Bonferroni post-hoc test). (C) Bright field image of a representative coronal section showing the location of the cannula placement in BLA (Cii) (anterior-posterior, $-3.12 \mathrm{~mm}$ from bregma [Paxinos and Watson 2007]; [*] injector site for SAL or MDZ infusions intra-BLA) and DH CA1 region (CI), where arrows indicate the spots of Dil injection. (D) Representative examples of apical dendritic segments of DH CA1 pyramidal neurons which were selected for quantitative analysis of dendritic spines from animals: SAL/S $(n=3$ rats), SAL/NS $(n=3), \mathrm{MDZ} / \mathrm{S}(n=4)$, and MDZ/NS $(n=3)$. Bar, $2 \mu \mathrm{m}$. $(E-G)$ Cumulative frequency of total $(E)$, mature $(F)$, and thin $(G)$ spine density on apical dendrites of hippocampal pyramidal cells CA1 stratum radiatum $(P<0.05$, by Kolmogorov-Smirnov test).

the dendrite to the tip of its head, $L$ ), the diameter of the neck (measured as the maximum neck diameter, $d_{\mathrm{n}}$ ), and the diameter of the head (measured as the maximum head diameter, $d_{\mathrm{h}}$ ) (Koh et al. 2002). Thus, individual spines were included in each category based on the specific ratios $L / d_{\mathrm{n}}$ and $d_{\mathrm{h}} / d_{\mathrm{n}}$. Stubby spines present an $L$ similar to the $d_{\mathrm{n}}$ and the $d_{\mathrm{h}}$, and in general the magnitude is $<1 \mu \mathrm{m}$. Mushroom spines present a $d_{\mathrm{h}}$ much larger than the $d_{\mathrm{n}}$ in which the $L$ is typically $<1 \mu \mathrm{m}$. Thin spines present an $L$ longer than $1 \mu \mathrm{m}$ that is much greater than the $d_{\mathrm{n}}$ (Koh et al. 2002; Tyler and Pozzo-Miller 2003; Calfa et al. 2012). Figure 1D shows a representative image for each particular dendritic spine classification.

A growing number of reports have shown that "stubby" and "mushroom" shaped dendritic spines, in virtue of their widespread $\mathrm{Ca}^{2+}$ transients in the parent dendrite and neighboring spines and because of the strength of the excitatory synapses formed on these spines (Harris 1999; Segal et al. 2000; Yuste et al. 2000; Nimchinsky et al. 2002; Kasai et al. 2003), are considered to be "mature" dendritic spines (Tyler and Pozzo-Miller 2003; Chapleau et al. 2009; Calfa et al. 2012). Thus, we have included the "stubby" and "mushroom" shaped dendritic spines in the definition of "mature" spines.

All the experimental protocols used in this work were approved by the Animal Care Committee of the Facultad de Ciencias Químicas, Universidad Nacional de Córdoba, which are consis- tent with the standards outlined in the NIH Guide for the Care and Use of Laboratory Animals.

All data collection was achieved in a blinded manner. Behavioral experiments were analyzed by a two-way ANOVA followed by Bonferroni post-hoc test or unpaired Student's $t$-test. Data were expressed as mean \pm SEM.

For the dendritic spines analysis, dendritic segments that belong to different slices from the same rat and from the same experimental group were considered for the statistical analysis. The distribution of the data does not rely on a normal distribution, and considering that mean values are rather insensitive to subtle changes, we use cumulative frequency plots to measure shifts in the total number of dendritic spines, mature dendritic spines, and thin dendritic spines per $10 \mu \mathrm{m}$ of dendritic segment in the different experimental groups. Cumulative distribution probabilities were compared by Kolmogorov-Smirnov tests (KS test). Data were also expressed as median (quartile) and compared by Kruskal-Wallis test. $P<0.05$ was considered statistically significant. We performed a compromise power analyses to determine the statistical power given the number of observations, sample means, and SD, using G*Power (Faul et al. 2007).

To analyze the behavioral performance during conditioning and posterior testing, animals were distributed among four groups for behavioral testing as follows: CA-noUS/NS ( $n=8$ rats), CAnoUS/S $(n=8)$, CA-US/NS $(n=8)$, and CA-US/S $(n=8)$ (Fig. 
1A). An unpaired Student's t-test on the percentage of time spent freezing during the pre-shock period showed a nonsignificant difference in freezing scores between the two groups, CA-US $6.88 \pm$ 0.62 (mean \pm SEM) vs. CA-noUS $7.08 \pm 0.7 \quad(P=0.566)$. The Student's $t$-test analysis for the post-shock period revealed that the percentage of time spent freezing of the CA-US group, $51.91 \pm 2.37$ (mean \pm SEM), was significantly higher than that of the CA-noUS, $15.73 \pm 2.65(P<0.001)$.

A two-way ANOVA for the freezing behavior during the testing session (Fig. 1B) indicated a significant effect of training $\left(F_{(1,32)}=125.95, P<0.001\right)$, stress $\left(F_{(1,32)}=69.082, P<0.001\right)$, and the interaction training $\times$ stress $\left(F_{(1,32)}=80.718, P<0.001\right)$. The Bonferroni post-hoc test revealed that the previous restraint experience enhanced freezing in trained rats in comparison to the rest of the groups $(P<0.001)$. Furthermore, the stress-induced promoting influence on fear retention was crucially dependent on the previous context-US association (Giachero et al. 2013).

To explore whether the combination of the conditioning procedure and the stress generates structural changes that would account for the increased fear memory, animals were subjected to a similar procedure and sacrificed for dendritic spine analyses $1 \mathrm{~d}$ after stress (Fig. 1A). Rats were distributed among four groups where spine counts were performed on a total of 115 dendritic segments as follows: CA-noUS/NS $(n=24$ segments, $933.07 \mu \mathrm{m}$ total dendritic length analyzed, three rats), CA-noUS/S $(n=29$ segments, $907.36 \mu \mathrm{m}$, three rats), CA-US/NS ( $n=30$ segments, $1076.01 \mu \mathrm{m}$, three rats), CA-US/S ( $n=32$ segments, $913.39 \mu \mathrm{m}$, three rats). For the total density of dendritic spines as well as for mature and thin dendritic spines, no significant differences were observed between rats from each particular experimental group comparing the results from the different dendritic segments ( $P>0.05, \mathrm{KS}$ test for all the comparisons). Figure 1C shows a representative image of DiI CA1 hippocampal labeling. Figure 1E shows representative examples of the dendritic segments in the stratum radiatum CA1 hippocampal area for each particular experimental group.

The analysis of the cumulative probability distributions for the total density of dendritic spines reflected a significant rightward shift toward higher numbers of total spines in CA-US/S animals in comparison to the rest of the experimental groups $(P<0.05$ for each individual comparison, Kolmogorov-Smirnov test) (Fig. 1F). This shift also resulted in a higher median (quartiles, total density $/ 10 \mu \mathrm{m})$ in CA-US/S $(16.9,14.4-18.8)$ with respect to the rest of the groups, CA-noUS/NS $(12.5,11.1-14.6)$, CAnoUS/S (13.5, 11.9-15.4), and CA-US/NS (13.1, 11.6-15.9) (Kruskal-Wallis test $=34.11, P<0.001)$.

In a similar way, a significant rightward shift toward higher numbers of mature dendritic spines in CA-US/S compared to the rest of the experimental groups was observed $(P<0.05$ for each individual comparison, Kolmogorov-Smirnov test) (Fig. $1 G)$. In parallel, a higher median (quartiles, mature spines/10 $\mu \mathrm{m})$ in CA-US/S $(13.5,10.7-15.1)$ was observed in comparison to CA-noUS/NS (9.1, 7.7-10.3), CA-noUS/S (9.1, 9.7-11.1), and CA-US/NS $(9.8,8-11.6)$ (Kruskal-Wallis test $=34.11, P<$ 0.001).

However, no significant differences were detected for thin dendritic spines between the experimental groups $(P>0.05$ for each individual comparison, Kolmogorov-Smirnov test) (Fig. $1 \mathrm{H})$. This also resulted in comparable median (quartiles, thin spines $/ 10 \mu \mathrm{m}$ ) between the experimental groups, CA-US/S (3.6, 2.7-4.5), CA-noUS/NS (3.8, 2.7-4.4), CA-noUS/S $(3.8,2.5-$ 4.5), and CA-US/NS $(3.8,2.3-4.8)$ (Kruskal-Wallis test $=0.143$, $P=0.986)$.

Overall, these results are indicative of a higher density of CA1 hippocampal dendritic spines, particularly the "mature" dendritic spines, as a result of the interaction between fear conditioning and stress.

Next, we examined the influence of intra-BLA MDZ (a positive modulator of GABAa sites) infusion prior to stress on both the fear retention and hippocampal dendritic remodeling generated by the combination of fear training and restraint. Fifty-three BLA cannulated animals were exposed to the CA and left undisturbed for a 3-min acclimation period (pre-shock period; mean \pm SEM of the percentage of time spent freezing, $1.8 \pm 1$ ), followed by a single unsignaled footshock ( $0.3 \mathrm{~mA}$, 3-sec duration). All animals were kept in the chamber for an additional $50 \mathrm{sec}$ (postshock period; mean \pm SEM of the percentage of time spent freezing, $44.8 \pm 7.4$ ). Twenty-four hours later, the animals were randomly distributed into four groups: SAL/S (trained animals that received an intra-BLA saline [SAL] administration $10 \mathrm{~min}$ prior to the stress session), SAL/NS (trained animals that received an intra-BLA SAL infusion and 10 min later were subjected to a brief handling without stressful manipulation), MDZ/S (trained animals that received an intra-BLA MDZ infusion $10 \mathrm{~min}$ prior to the stress session), and MDZ/NS (trained animals that received an intra-BLA MDZ infusion 10 min prior to a brief handling without stressful manipulation) (Fig. 2A). The next day, 32 cannulated animals (eight animals for each experimental group) were tested for their freezing behavior during re-exposure to the CA. A twoway ANOVA for the freezing behavior indicated a significant effect of treatment $\left(F_{(1,28)}=20.68, P=0.0001\right)$, stress $\left(F_{(1,28)}=23.75\right.$, $P=0.00004)$, and treatment $\times$ stress interaction $\left(F_{(1,28)}=13.99\right.$, $P=0.00084$ ) (Fig. 2B). The Bonferroni post-hoc test revealed that stressed animals with intra-BLA SAL exhibited a higher percentage of freezing compared to the rest of the experimental groups $(P>$ $0.05)$. This result indicates that MDZ intra-BLA infusion prevented the promoting influence of stress on the resulting fear memory.

For spine analysis, the rest of the cannulated animals $(n=21)$ were randomly distributed into the following groups where spine counts were performed on a total of 161 dendritic segments: SAL/ S $(n=34$ segments, $1141.06 \mu \mathrm{m}$ of total dendritic length analyzed, five rats), SAL/NS (38 segments, $1219.62 \mu \mathrm{m}$, five rats), MDZ/S (46 segments, $1549.06 \mu \mathrm{m}$, six rats), and MDZ/NS (34 segments, $1422.99 \mu \mathrm{m}$, five rats) (Fig. 2A,Ci).

For the total density of dendritic spines as well as for mature and thin dendritic spines, no significant differences were observed between rats from each particular experimental group comparing the results from the different dendritic segments $(P>0.05$, KS test for all the comparisons).

The analysis of the cumulative probability distributions for the total density of dendritic spines reflected a significant rightward shift, toward higher numbers of dendritic spines in SAL/S animals in comparison to the rest of the experimental groups $(P<0.05$ for each individual comparison, Kolmogorov-Smirnov test) (Fig. 2E). Curiously, MDZ/S animals presented a significant left shift, toward fewer numbers of dendritic spines, in comparison to SAL/NS animals (Kolmogorov-Smirnov test, KSZ, 1.3945, $P=0.0409$ ). The shift toward higher numbers of dendritic spines in SAL/S also resulted in a higher median (quartiles, total density/ $10 \mu \mathrm{m}), 16.2(15.3-17.5)$, with respect to the rest of the groups, SAL/NS (12.7, 11.1-13.7), MDZ/S (11.5, 10.1-12.7), and MDZ/ NS $(12.1,11.4-12.8)$ (Kruskal-Wallis test $=63.646, P<0.001)$.

The higher number of the total dendritic spines in SAL/S animals was also evident for mature dendritic spines. We observed a significant rightward shift toward higher numbers of mature dendritic spines in SAL/S compared to the rest of the experimental groups $(P<0.05$ for each individual comparison, KolmogorovSmirnov test) (Fig. 2F). Similarly, a higher median (quartiles, mature spines $/ 10 \mu \mathrm{m})$ in SAL/S $(11.5,9.6-13.5)$ was observed in comparison to SAL/NS (8.4, 6.8-9.4), MDZ/S (7.9, 7.1-9.2), and MDZ/NS $(7.9,6.8-9.4)($ Kruskal-Wallis test $=48.802, P<0.001)$. 
The analysis of thin dendritic spines resulted in a significant left shift toward fewer thin spines in MDZ/S in comparison to SAL/S animals $(P=0.0042$, Kolmogorov-Smirnov test, $K S Z$, 1.754) (Fig. 2G) and SAL/NS animals $(P=0.007$, KolmogorovSmirnov test, $K S Z, 1.674$ ) (Fig. $2 \mathrm{G}$ ). No significant differences were detected for the rest of the experimental groups $(P>0.05$ for each individual comparison, Kolmogorov-Smirnov test) (Fig. 2G). The same significant effect was also evident in the median (quartiles, thin spines $/ 10 \mu \mathrm{m}$ ) of the different experimental groups, SAL/S (4.4, 3.6-5.6), SAL/NS (4, 3.5-5.1), MDZ/S (3.3, 2.1-4.3), MDZ/NS (4.2, 2.9-4.9) (Kruskal-Wallis test $=12.024$, $P=0.0073)$.

Overall, these findings suggest that MDZ intra-BLA prior to stress prevented the enhancement of the density of "mature" spines elicited by the combination of fear conditioning and stress.

The present results revealed a robust fear memory in the trained animals that later experienced an unrelated stressful event. This memory trace was reported elsewhere to be persistent (Giachero et al. 2013) and crucially dependent on the previous context-US association. In addition, previous data revealed that this facilitating action was not due to an unspecific generalization of fear or to a sensitized response to restraint resulting from a nonassociated past-footshock experience (Giachero et al. 2013).

The facilitated fear contextual representation following the combination of conditioning and stress was associated with a consistent increase of the total number of dendritic spines in $\mathrm{DH}$, particularly of the functional "mature" spines. In contrast, neither the fear conditioning procedure nor the threatening event per se was able to elicit this structural remodeling in $\mathrm{DH}$. In agreement, previous reports have shown that a strong memory trace is accompanied by a higher number of dendritic spines in various learning and memory paradigms, including fear conditioning (Moser et al. 1994; Leuner et al. 2003; Restivo et al. 2009). Moreover, the remodeling of dendritic spines has been suggested to be involved in synaptic plasticity (Trommald et al. 1996; Maletic-Savatic et al. 1999; Bastrikova et al. 2008; Yang et al. 2008), which further emphasizes the functional relevance of dendritic spine changes on memory formation.

Consistent with previous findings (Giachero et al. 2013), intra-BLA MDZ prevented the augmented fear retention following stress in conditioned rats. Interestingly, and in support of the behavioral findings, the stimulation of the GABAa sites in BLA impeded the structural remodeling changes, particularly in mature spines, since MDZ stressed animals presented a similar number of total and mature spines as unstressed rats. These data suggest that both phenomena (contextual fear memory and remodeling of mature spines in $\mathrm{DH}$ ) are interrelated. Despite the fact that $\mathrm{MDZ}$ also prevented the changes in total spines in stressed rats, it is noticeable that MDZ stressed animas exhibited a lower number of total spines than saline unstressed animals. Similarly, MDZ stressed rats presented a reduced number of thin-shaped dendritic spines as compared to SAL stressed and unstressed animals, suggesting that the MDZ-induced effect is even more pronounced on thin spines regardless of the stress condition. The explanation of such an effect is unclear and future research will need to address this topic in more detail.

Overall, it is relevant to indicate that the promoting influence of stress on fear memory and on synaptic remodeling in hippocampal mature spines is regulated by the GABAergic transmission in BLA.

In line with this view, the enhancement of hippocampal Arc expression, a protein involved in synaptic transmission and plasticity (Bramham et al. 2008, 2010), after fear conditioning was attenuated by the intra-BLA infusion of a GABAa agonist (Huff et al. 2006). Furthermore, muscimol infusion into the BLA blocks stress-induced impairments in hippocampal long-term potentia- tion and spatial memory (Kim et al. 2005). Hence, there is growing evidence that the amygdala has a prominent role in the influence of stress on hippocampal function (Kim et al. 2005; Ghosh et al. 2013). To sum up, these findings highlight the key role played by BLA in coordinating structural plasticity in the downstream areas involved in memory processing and storage (McIntyre et al. 2003; McGaugh 2004).

In conclusion, structural remodeling in DH accompanies the facilitated fear memory following conditioning and aversive stimulation. In addition, this stress-induced promoting influence is under the modulation of a GABA-dependent mechanism in BLA.

\section{Acknowledgments}

M.G. thanks CONICET. This research was supported by grants from SECYT-Universidad Nacional de Córdoba to V.A.M. and G.D.C., CONICET and Agencia Nacional de Promoción Científica y Tecnológica-FONCYT (Argentina) to V.A.M., and IBRO Return Home Fellowship to G.D.C. We thank Lorena Mercado, Estela Salde, Cecilia Sampedro, and Carlos Mas for technical assistance and Paul Hobson for English technical assistance.

\section{References}

Bastrikova N, Gardner GA, Reece JM, Jeromin A, Dudek SM. 2008. Synapse elimination accompanies functional plasticity in hippocampal neurons. Proc Natl Acad Sci 105: 3123-3127.

Beylin AV, Shors TJ. 1998. Stress enhances excitatory trace eyeblink conditioning and opposes acquisition of inhibitory conditioning. Behav Neurosci 112: 1327-1338.

Boda B, Alberi S, Nikonenko I, Node-Langlois R, Jourdain P, Moosmayer M, Parisi-Jourdain L, Muller D. 2004. The mental retardation protein PAK3 contributes to synapse formation and plasticity in hippocampus. J Neurosci 24: 10816-10825.

Bramham CR, Worley PF, Moore MJ, Guzowski JF. 2008. The immediate early gene arc/arg3.1: Regulation, mechanisms, and function. J Neurosci 28: 11760-11767.

Bramham CR, Alme MN, Bittins M, Kuipers SD, Nair RR, Pai B, Panja D, Schubert M, Soule J, Tiron A, et al. 2010. The Arc of synaptic memory. Exp Brain Res 200: $125-140$.

Calfa G, Chapleau CA, Campbell S, Inoue T, Morse SJ, Lubin FD, Pozzo-Miller L. 2012. HDAC activity is required for BDNF to increase quantal neurotransmitter release and dendritic spine density in CA1 pyramidal neurons. Hippocampus 22: 1493-1500.

Chapleau CA, Pozzo-Miller L. 2007. Activity-dependent structural plasticity of dendritic spines. In Molecular mechanisms of memory (ed. Sweatt JD), Vol. 4, pp. 695-720. Elsevier, Oxford.

Chapleau CA, Calfa GD, Lane MC, Albertson AJ, Larimore JL, Kudo S, Armstrong DL, Percy AK, Pozzo-Miller L. 2009. Dendritic spine pathologies in hippocampal pyramidal neurons from Rett syndrome brain and after expression of Rett-associated MECP2 mutations. Neurobiol Dis 35: 219-233.

Cordero MI, Venero C, Kruyt ND, Sandi C. 2003. Prior exposure to a single stress session facilitates subsequent contextual fear conditioning in rats. Horm Behav 44: 338-345.

Fanselow MS, Dong HW. 2010. Are the dorsal and ventral hippocampus functionally distinct structures? Neuron 65: 7-19.

Faul F, Erdfelder E, Lang AG, Buchner A. 2007. G*Power 3: A flexible statistical power analysis program for the social, behavioral, and biomedical sciences. Behav Res Methods 39: 175-191.

Ghosh S, Laxmi TR, Chattarji S. 2013. Functional connectivity from the amygdala to the hippocampus grows stronger after stress. J Neurosci 33: $7234-7244$.

Giachero M, Bustos SG, Calfa G, Molina VA. 2013. A BDNF sensitive mechanism is involved in the fear memory resulting from the interaction between stress and the retrieval of an established trace. Learn Mem 20: 245-255.

Gray EG. 1959. Electron microscopy of synaptic contacts on dendrite spines of the cerebral cortex. Nature 183: 1592-1593.

Harris KM. 1999. Structure, development, and plasticity of dendritic spines. Curr Opin Neurobiol 9: 343-348.

Huff NC, Frank M, Wright-Hardesty K, Sprunger D, Matus-Amat P, Higgins E, Rudy JW. 2006. Amygdala regulation of immediate-early gene expression in the hippocampus induced by contextual fear conditioning. J Neurosci 26: 1616-1623. 
Kasai H, Matsuzaki M, Noguchi J, Yasumatsu N, Nakahara H. 2003. Structure-stability-function relationships of dendritic spines. Trends Neurosci 26: 360-368.

Kim JJ, Fanselow MS. 1992. Modality-specific retrograde amnesia of fear. Science 256: $675-677$.

Kim JJ, Koo JW, Lee HJ, Han JS. 2005. Amygdalar inactivation blocks stress-induced impairments in hippocampal long-term potentiation and spatial memory. J Neurosci 25: 1532-1539.

Koh IY, Lindquist WB, Zito K, Nimchinsky EA, Svoboda K. 2002. An image analysis algorithm for dendritic spines. Neural Comput 14: 1283-1310.

LeDoux JE. 2000. Emotion circuits in the brain. Annu Rev Neurosci 23: $155-184$.

Leuner B, Falduto J, Shors TJ. 2003. Associative memory formation increases the observation of dendritic spines in the hippocampus. J Neurosci 23: 659-665.

Maldonado NM, Martijena ID, Molina VA. 2011. Facilitating influence of stress on the consolidation of fear memory induced by a weak training: Reversal by midazolam pretreatment. Behav Brain Res 225: 77-84.

Maletic-Savatic M, Malinow R, Svoboda K. 1999. Rapid dendritic morphogenesis in CA1 hippocampal dendrites induced by synaptic activity. Science 283: $1923-1927$.

Maren S, Fanselow MS. 1997. Electrolytic lesions of the fimbria/fornix, dorsal hippocampus, or entorhinal cortex produce anterograde deficits in contextual fear conditioning in rats. Neurobiol Learn Mem 67: $142-149$.

McGaugh JL. 2004. The amygdala modulates the consolidation of memories of emotionally arousing experiences. Annu Rev Neurosci 27: 1-28.

McIntyre CK, Power AE, Roozendaal B, McGaugh JL. 2003. Role of the basolateral amygdala in memory consolidation. Ann N Y Acad Sci 985: $273-293$.

Moser MB, Trommald M, Andersen P. 1994. An increase in dendritic spine density on hippocampal CA1 pyramidal cells following spatial learning in adult rats suggests the formation of new synapses. Proc Natl Acad Sci 91: $12673-12675$.

Murphy DD, Segal M. 1996. Regulation of dendritic spine density in cultured rat hippocampal neurons by steroid hormones. J Neurosci 16: 4059-4068.

Nimchinsky EA, Sabatini BL, Svoboda K. 2002. Structure and function of dendritic spines. Annu Rev Physiol 64: 313-353.

Paxinos G, Watson C. 2007. The rat brain in stereotaxic coordinates. Academic Press, San Diego.

Phillips RG, LeDoux JE. 1995. Lesions of the fornix but not the entorhinal or perirhinal cortex interfere with contextual fear conditioning. J Neurosci 15: 5308-5315.
Pozzo-Miller LD, Inoue T, Murphy DD. 1999. Estradiol increases spine density and NMDA-dependent $\mathrm{Ca}^{2+}$ transients in spines of CA1 pyramidal neurons from hippocampal slices. J Neurophysiol

81: $1404-1411$.

Restivo L, Vetere G, Bontempi B, Ammassari-Teule M. 2009. The formation of recent and remote memory is associated with time-dependent formation of dendritic spines in the hippocampus and anterior cingulate cortex. J Neurosci 29: $8206-8214$.

Rodriguez A, Ehlenberger DB, Dickstein DL, Hof PR, Wearne SL. 2008 Automated three-dimensional detection and shape classification of dendritic spines from fluorescence microscopy images. PloS One 3: e1997.

Rodriguez Manzanares PA, Isoardi NA, Carrer HF, Molina VA. 2005. Previous stress facilitates fear memory, attenuates GABAergic inhibition, and increases synaptic plasticity in the rat basolateral amygdala. J Neurosci 25: 8725-8734.

Segal I, Korkotian I, Murphy DD. 2000. Dendritic spine formation and pruning: Common cellular mechanisms? Trends Neurosci 23: $53-57$.

Shors TJ. 2001. Acute stress rapidly and persistently enhances memory formation in the male rat. Neurobiol Learn Mem 75: $10-29$.

Shors TJ, Weiss C, Thompson RF. 1992. Stress-induced facilitation of classical conditioning. Science 257: 537-539.

Trommald M, Hulleberg G, Andersen P. 1996. Long-term potentiation is associated with new excitatory spine synapses on rat dentate granule cells. Learn Mem 3: 218-228.

Tyler WJ, Pozzo-Miller L. 2003. Miniature synaptic transmission and BDNF modulate dendritic spine growth and form in rat CA1 neurones. J Physiol 553: 497-509.

Vetere G, Restivo L, Cole CJ, Ross PJ, Ammassari-Teule M, Josselyn SA, Frankland PW. 2011. Spine growth in the anterior cingulate cortex is necessary for the consolidation of contextual fear memory. Proc Natl Acad Sci 108: $8456-8460$.

Yang Y, Wang XB, Frerking M, Zhou Q. 2008. Spine expansion and stabilization associated with long-term potentiation. J Neurosci 28: $5740-5751$.

Yuste R, Majewska A, Holthoff K. 2000. From form to function: Calcium compartmentalization in dendritic spines. Nat Neurosci 3: $653-659$.

Received May 6, 2013; accepted in revised form August 5, 2013. 


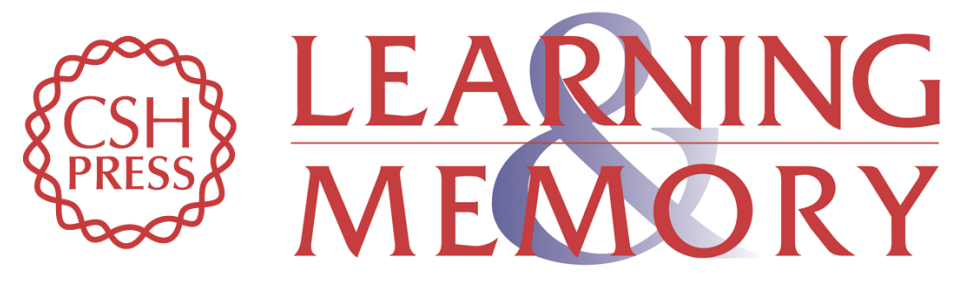

\title{
Hippocampal structural plasticity accompanies the resulting contextual fear memory following stress and fear conditioning
}

\author{
Marcelo Giachero, Gaston D. Calfa and Victor A. Molina
}

Learn. Mem. 2013, 20:

Access the most recent version at doi:10.1101//m.031724.113

\begin{aligned} & \hline References $\begin{array}{l}\text { This article cites } 42 \text { articles, } 19 \text { of which can be accessed free at: } \\ \text { http://learnmem.cshlp.org/content/20/11/611.full.html\#ref-list-1 }\end{array} \\ & \begin{aligned} \text { Creative } \\ \text { Commons } \\ \text { License }\end{aligned} \begin{array}{l}\text { This article is distributed exclusively by Cold Spring Harbor Laboratory Press for the } \\ \text { first } 12 \text { months after the full-issue publication date (see } \\ \text { http://learnmem.cshlp.org/site/misc/terms.xhtml). After } 12 \text { months, it is available under } \\ \text { a Creative Commons License (Attribution-NonCommercial } 3.0 \text { Unported), as } \\ \text { described at http://creativecommons.org/licenses/by-nc/3.0/. }\end{array} \\ & \begin{array}{c}\text { Receive free email alerts when new articles cite this article - sign up in the box at the } \\ \text { top right corner of the article or click here. }\end{array} \\ & \begin{array}{c}\text { Service } \\ \text { Serting }\end{array}\end{aligned}$

